\title{
Formulation of FISPLAN: A Fuzzy Logic based Reactive Planner for AUVs towards Situation Aware Control
}

\author{
Subhra Kanti Das \\ Robotics \& Automation, CSIR-CMERI, M G Avenue, Durgapur, WB, INDIA, PIN-713209 \\ E-mail: subhrakanti.das82@gmail.com \\ Dibyendu Pal \\ Robotics \& Automation, CSIR-CMERI, M G Avenue, Durgapur, WB, INDIA, PIN-713209 \\ E-mail: subhrakanti.das82@gmail.com
}

\begin{abstract}
- the paper presents a detailed discussion on the structural organisation of a Fuzzy Inference System Planner (FISPLAN) for Autonomous Underwater Vehic les (AUVs), including elaboration of membership functions for the inputs as well as outputs. The inference mechanism is detailed with discussions on the rule base, which in essence incorporates the planning logic. In order to assess the effectiveness of the planner as a means of reactive escape under critical situations, a case study is studied with reference to a state of the art AUV. An approximate subsea current model is developed from field observations, and residual energy is estimated by referring to a typical Lithium-polymer cell discharge characteristic together with data recorded in actual field trials. Situations are simulated by considering different combinations of sea-currents as well as status of resident energy. Results reveal that the simu lated system, by virtue of the planner, is capable of perceiving situations, thereby realizing their imminence and making a decisive action thereupon. In concise, the fuzzy planner may be considered to provide human-like perception of situations on the basis of crisp observations. Furthermore dynamics of the system are modelled with actual parameters, and subsequently controller responses for pitching and velocity correction are illustrated. Choice of planning interval is also expressed as a function of the controllers' response.
\end{abstract}

Index Terms - Planning, Reactive Architecture, Fu zzy, Situation Awareness, Escape

\section{Introduction}

Over the past few decades, an increasing demand of oceanographic explorations have given rise to the development of technologies particularly suited for unmanned underwater operations, out of which AUVs have framed out a niche as the most cost-effective and sustainable technology over the coming years. AUVs stand for autonomous underwater vehicles. These are mobile robotic systems, which can operate underwater at great depths (ranging from 150 to 6000 meters) with limited human intervention or supervis ory control [1]. The criticality of operation of such self-controlled systems lies in the design of robust software architecture, involving both planning and control existing in the form of a set of well-coordinated functional modules interfacing with various sensors, actuators and associated controllers present in the system. Although for most practical cases, control and planning modules lie diffused existing as a single entity, it is significant enough to go for both structural and functional decomposition of the processes into mutually cooperative and individually identifiable components. Originating from this underlying concept several diversified architectures have been proposed and worked out in the literature. However, most of them can be categorized into (1) reactive [2]-[5] (2) deliberative [6]-[8] as well as the recently formulated (3) hybrid deliberative and reactive [9], [10], [11], [12]. Contrastingly, few works [13], [14] have incorporated probabilistic planning in order to achieve the mission goals in a completely non-deterministic environment. The proposed approach centers on the formulation of the navigation problem as Partially Observable Markov Decision Problem (POMDP). This inherently reinforces the control frame work towards dynamic uncertainties of the environment. However, as a still more recent approach, a new concept of situation awareness with semantic representation, has been introduced [15] with the idea that heterogeneous real-world data of very different type must be processed by and run through several different layers, to be finally available in a suited format and at the right place to be accessible by high-level decision-making agents.

The rest of the document is organized as follows -the subsequent section presents a detailed discussion on the structural organization of the Fuzzy planner. Membership functions are elaborated and the rule base is dissected for enforcement of the planning logic. The 
following section involves a case study with reference to a state of the art AUV, targeted towards formulating an approximate subsea current model and computing residual energy from a typical Lithium-polymer cell characteristic. The last section details simulation conditions and results obtained thereof. Results reveal that the simulated system is capable of translating observations into human understandable situation and making a decisive action thereupon. The es cape actions are well exhibited in a dynamic situation, where in seacurrents as well as residual energy are shown to be changing probabilistically over time.

\section{Proposed Approach for Planning}

In the present context of the paper, an attempt has been made to present a fuzzy logic based reactive planning frame work (FISPLAN), supported by dynamic situation assessment as a means of situation aware decision making process for AUVs. Conceptually, situation awareness is a means of representing perception about the environment to the vehicle regarding the available observations [16], so that decisions can be made about the uncertain future. Informally, situations are seen as states of a dynamic system observed at particular time; Complexity of the situations may range from a single attribute value of an object, to complex collections of objects interlinked by various relations. A careful scrutiny of a typical mission shall reveal the indispensable requirement for situation awareness for AUVs during operations-since AUVs have the capability of operating practically at any depth, more often than not they are prone to be faced with subsea currents. Usually surface currents are more predictable with prior information (usually from weather forecasts) than subsea currents, which are erratic abrupt and to the greatest extent uncertain. This can lead to a very common situation where in the AUV facing a strong subsea current doesn't actually make the desired motion, with an almost expendable drainage of energy. A human-like solution in this regard is to escape the current operational depth, and push up to a safer depth of operation followed by depth-sensitive reconfigurations and readjustments. This however, is in close connection to the fact that subsea currents can vary abruptly with depths. Moreover, observation over the residual energy available with the system may lead to the understanding (from the perspective of the system) of permis sibility for carry ing out such escape operations Situations like these are analytically quite intractable owing to the infeasibility in exactly resolving the uncertain dynamics of the subsea environment. Moreover, the inherent difficulty in computing the relation between magnitude of sea-current and the escape operations further complicates the problem. On the contrary, transformation of the situation as sessment in human realizable form and mode of behavior can be an effective means. Hence the proposed planning architecture is based upon Fuzzy logic, which fuzzifies the inputs-sea current and residual energy, infers on the basis of a set of rules and generates an escape action in terms of pitch and linear velocity. This shall enable the AUV to escape to a lower depth having a lower magnitude of sea-currents. By virtue of the proposed planning, the system eventually attains situational autonomy, which is a purely reactive phenomenon and hence the underlying concept can be quite effective in formulating a situation aware mission management framework for an AUV.

\section{FISPLAN: Fuzzy Inference System Planner}

Originally advocated by Zadeh [17] and Mamdani and Assilian [18], fuzzy logic has become a mean of collecting human knowledge and experience and dealing with uncertainties in the control process. Fuzzy logic provides a foundation for approximate reasoning by extending traditional Boolean logic and by allowing truth to be a matter of degree as in the case of membership degree in fuzzy sets. It is applicable to problems where linguistic expression is significant than numbers. In the present section the essential planning architecture shall be expressed through the design of a Fuzzy Inference System(FIS).

\subsection{Fuzzy Inference System}

The FIS is designed as a two input, two output Mamdani system as depicted in fig 1 . Sea current and residual energy are the inputs, for which crisp observations are available. Fuzzification is carried out and fuzzified outputs are generated on the basis of a rule engine which forms the kernel of the FIS. Subsequently defuzzification is being carried out with the centre-of-area (COA) method.

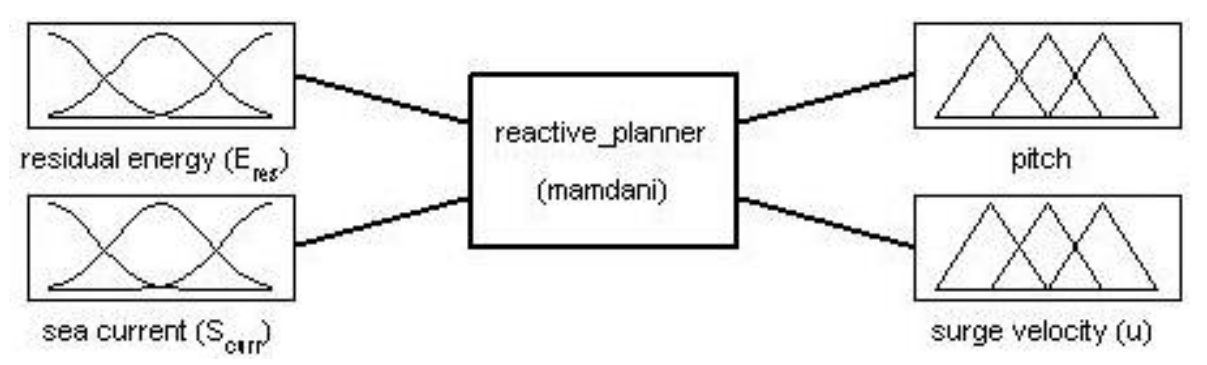

Fig. 1: Skeletal framework of the Fuzzy Planner 


\subsection{FISPLAN Inputs and Their Membership Functions}

Each of the inputs is represented in the form of two triangular and one trapezoidal membership functions. The membership classification for the inputs is as follows:

1. Sea Current: (i) weak currents between 0 and 10 knots, (ii) medium currents between 2.5 and 17.5 knots, and (iii) strong currents between 10 and 20 knots.

2. Residual Energy: (i) critical (CRT) with energy less than $50 \%$, (ii) a larming (ALM) with energy between $25 \%$ and $75 \%$, and (iii) safe (SFE) with energy more than $50 \%$ up to $100 \%$.

The membership functions as defined above are illustrated in Fig 2. The overlap between membership functions has been kept at 0.5 for a heuristically optimal performance of the FIS. At this point, it may be noted that the nonlinearities of the inputs are represented as piecewise linear segments.
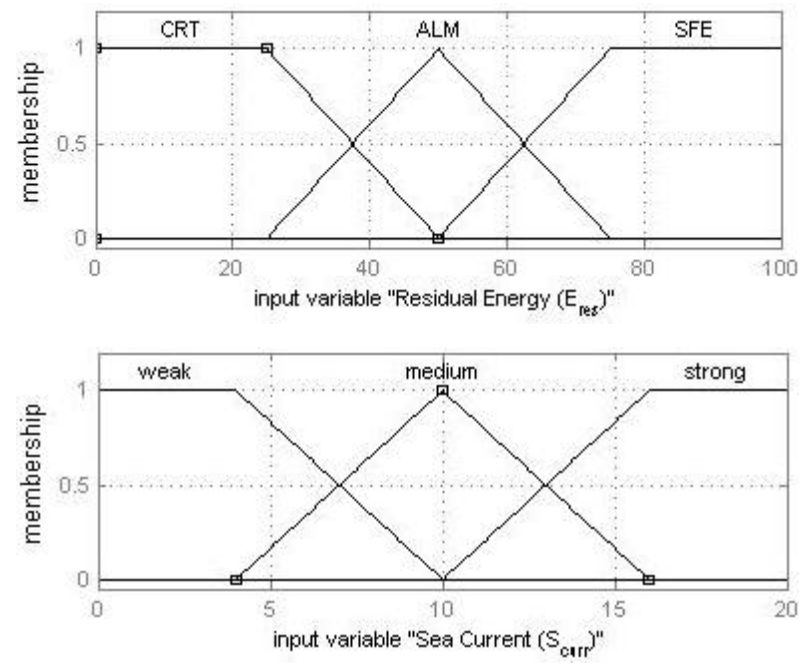

Fig. 2: FIS inputs and membership functions

\subsection{FISPLAN Outputs and Their Membership Functions}

Similar to the inputs, the FIS outputs as shown in fig 3 are represented as follows:

1. Pitch: (i) low pitch for less than 0.2 radian, (ii) moderate pitch between 0.1 and 4.5 radian, and (iii) high pitch between 0.35 and 5radian.

2. Surge Velocity: (i) Gaussian membership function with $\mu=0.2, \sigma 2=0.5945$ for low velocities, and (ii) Gaussian membership function with $\mu=3.0$, $\sigma 2=0.5945$ for high velocities.

Contrastingly however, the surge velocity is represented as continuously varying nonlinear functions, so that a greater dynamic variability of motion can be maintained for the vehicle.

\subsection{FISPLAN Rule Base}

The effectiveness of FIS design resides both on the judicious classification of inputs and outputs as well as soundness of the rule-base. Fuzzy rules are a set of human conceivable formulae each consisting of an antecedent and a consequent involving the fuzzy inputs and outputs. In the proposed FIS framework the rules have been formulated with a view to incorporate situation assessment including uncertainty in decision making. A snapshot of the triggering of various rules in the engine is illustrated in Fig 4. Rules can be formulated if we can appreciate the various levels of inference drawn for the proposed FIS. Incidentally, for a good level of resident energy available to the system, the planner can take decision reactively in response to the magnitude of the sea-current. Hence for low currents it may not appreciable change the present course of action. For mediu m currents it can prescribe a gradual escape to a lesser depth by combining a medium pitching with a moderate velocity along the surge axis. However, for strong currents, the planner may decide upon quickly escaping to lesser depths so that minimal energy is spent in fighting against the currents. The above discussion so far can summarily lead to the formulation of the following rules:
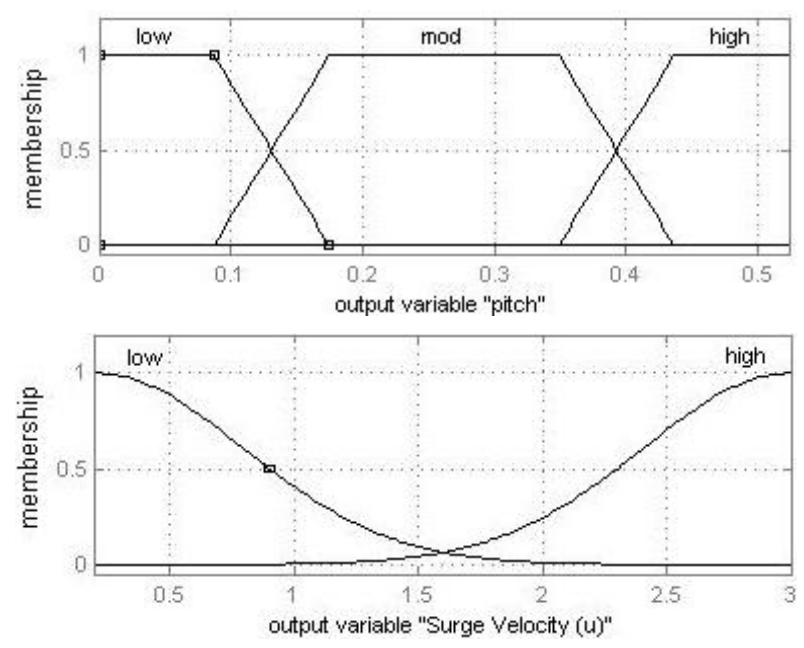

Fig. 3: FIS outputs and membership functions

1. If (Eres is SFE) and (Scurr is weak) then (pitch is low) and ( $u$ is low)

2. If (Eres is SFE) and (Scurr is medium) then (pitch is mod) and ( $u$ is low)

3. If (Eres is SFE) and (Scurr is strong) then (pitch is high) and (u is high)

4. If ( $E_{\text {res }}$ is $\left.A L M\right)$ and ( $S_{\text {curr }}$ is weak) then (pitch is low) and ( $u$ is low) 
5. If ( $E_{\text {res }}$ is $\left.A L M\right)$ and ( $S_{\text {curr }}$ is medium) then (pitch is mod) and (u is low)

6. If ( $E_{\text {res }}$ is ALM) and ( $S_{\text {curr }}$ is strong) then (pitch is high) and (u is low)

7. If ( $E_{\text {res }}$ is CRT) and ( $S_{\text {curr }}$ is weak) then (pitch is low) and (u is low)
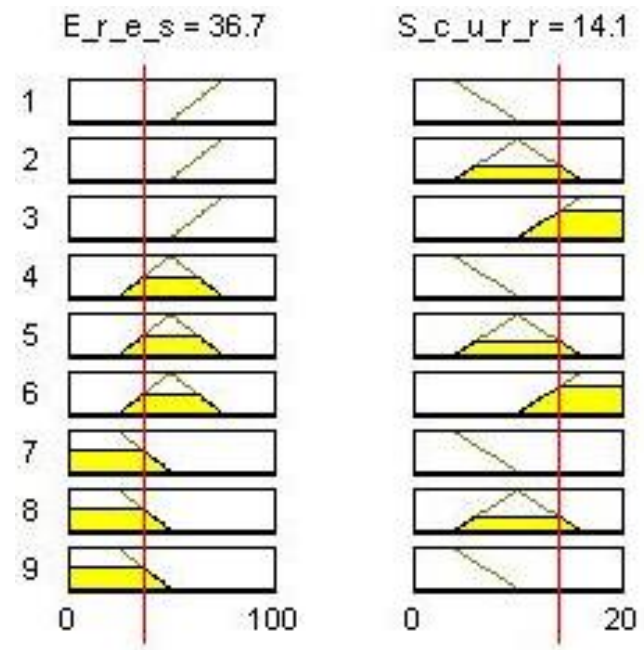

Fig. 4: Rule View showing a snapshot of how the rules get triggered for a given set of inputs

Contrastingly, as for a human driver, the fuzzy planner also puts the residual energy at a higher priority than sea-currents. In this context it may be noted that, propulsion is usually a more energy intensive issue than orientation (including pitching). As a result, in situations of low resident energy, the planner should take a decision in order to optimise reactive escape and sufficient sustenance of life after escape so that the system can safely surface if not complete the mission. Hence, the planner triggers a low velocity for ALM and CRT levels of resident energy. Strong and medium currents shall be dealt with high and moderate pitching for ALM and CRT energy levels. Strikingly however, the rule base suggests a high pitching for medium currents during the observation of CRT energy level. On the basis of this understanding of the situation handling capabilities of the planner the following rules can be formulated:

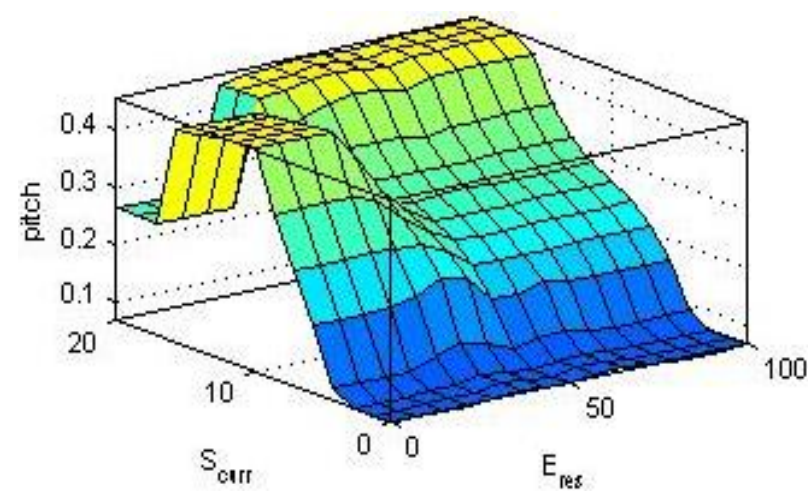

8. If ( $E_{\text {res }}$ is $\left.C R T\right)$ and ( $S_{\text {curr }}$ is medium) then (pitch is high) and (u is low)

9. If ( $E_{\text {res }}$ is $\left.C R T\right)$ and ( $S_{\text {curr }}$ is weak) then (pitch is low) and ( $u$ is low)
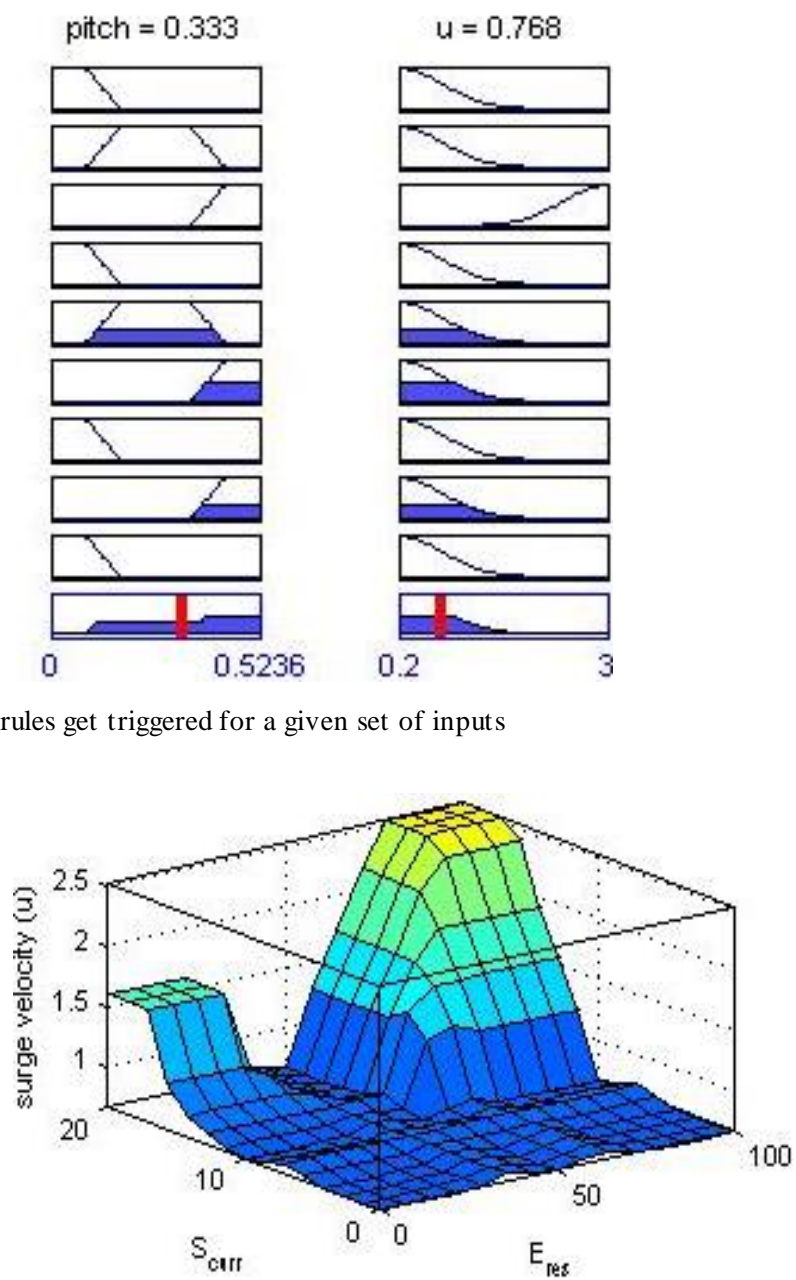

Fig. 5: Rule Surface illustrating the continuously varying gradient of pitch (top) and surge velocity (bottom) over a set of continuously varying magnitudes of sea current and estimate of residual energy

Fig. 5 illustrates the dynamic variations in surge velocity as well as pitching for continuously varying inputs of sea currents and residual energy. The velocity is low in the quadrants where residual energy is less than $50 \%$ irrespective of the strength of sea-currents, which is reflective of the capability of the planner to interpret and assess the criticality of situations from crisp observations. The following section details a case study involving the dynamic model of an actual AUV prototype as well as environmental parameters formulated from the observations of the vehicle during field experimentation. 


\section{Case Study}

Case study involves a state-of-the-art unmanned underwater vehicle AUV-150 [19], [20] developed by CSIR-CMERI, India and tested for an operational depth of 150 meters. Data recorded during the sea-trials of the same however revealed certain interesting findings about various environmental parameters with sub-sea currents in particular. Sea currents have been recorded by an acoustic Doppler current profiler (ADCP) physically attached with the system. Data has been logged in real-time at various depths of operation starting from the surface up to 150 meters. Residual energy has been recorded in real time by a dedicated power management system. In the present context of the paper, the recorded data is being utilized in order to formulate the environment model for sea-currents as well as state of energy. The attempt is significant from the perspective of conjuring up a realistic situation. Offline simulation of the same can help in establishing the efficacy of the planner, which shall be discussed in details in the subsequent section. The present section shall detail modelling of the system dynamics for simulating actuation behaviour of the vehic le as a result of the planner's prescribed actions; approximate model of sea-currents and estimation of residual energy using Lithium Polymer cells.

\subsection{System Model}

AUV-150 is a nonlinear 6-DOF system as illustrated in fig 6. Design specific roll stabilization has been achieved using two sets of four static fins. This further reduces the overall degrees of independent control to 5 . Notwithstanding the 5-DOF controllability of the system, we consider the set of horizontal thrusters for yaw control alone and not for sway. The dynamic state of the vehicle is expressed as $[u, w, \psi, \theta]$, where $u$ and $w$ are respectively surge and heave velocities in body frame. $\theta$ and $\psi$ are respectively the pitch and yaw angles of the vehicle. Considering the 6-DOF nonlinear dynamics model as defined in [21], the underlying dynamics associated with the various motions of AUV150 may be expressed as follows:

$$
M \times(d v / d t)+C(v) v+D(v) v+g(\eta)=\tau
$$

Where $C(v)$ and $D(v)$ are Coriolis and Damping terms.

Table 1: Values for the hydrodynamic mass variables

\begin{tabular}{|c|c|c|}
\hline Coefficients & Meanings & Values \\
\hline $\mathrm{Xu}$ & Hydrodynamic mass along X & $48.8 \mathrm{~kg}$ \\
\hline Iyy & Moment of Inertia about Y & $8.05 \times 10-2 \mathrm{~kg}-\mathrm{m} 2$ \\
\hline $\mathrm{MR}$ & Rigid Body Mass & $484 \mathrm{~kg}$ \\
\hline
\end{tabular}

The design speed of AUV-150 is estimated to be close to 4 Knots (i.e. $2 \mathrm{~m} / \mathrm{sec}$ ), as a result of which the Coriolis and gravitational terms are computed to be negligible. Thus ignoring theses terms the effective model may be defined as:

$$
M \times(d v / d t)+D(v) v=\tau
$$

$\mathrm{M}$ is computed as the total hydrodynamic mass and is mathematically defined as:

$$
M=M_{R}+M_{A}
$$

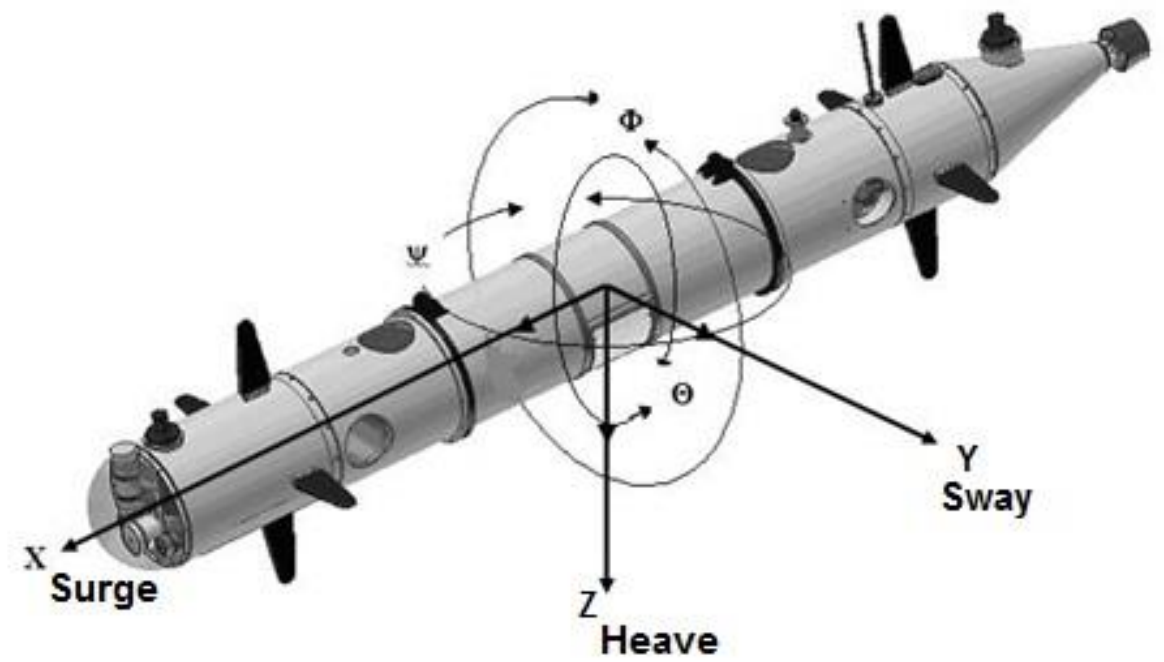

Fig. 6: 3D visualization of an AUV model reflecting 6 DOF in body-fixed as well as earth-fixed reference frame

The former being rigid body mass of the vehicle and the latter being hydrodynamic added mass attributed with motion of the vehicle. In the present context of the paper, values are expressed for the hydrodynamic mass variables along the surge (forward motion) and about the sway axes (for pitching). Table I en lists the values of $X_{u}, I_{y y}$ and $M_{R}$. 
Table 2: Sea currents as distributed normally and as a function of depths

\begin{tabular}{|c|c|c|}
\hline Depth Range & Mean $(\boldsymbol{\mu})$ & Variance $(\boldsymbol{\sigma 2})$ \\
\hline $0-25$ & 0.38 & 0.057 \\
\hline $25-50$ & 0.26 & 0.005 \\
\hline $50-75$ & 0.47 & 0.0483 \\
\hline $75-100$ & 4.28 & 3.23 \\
\hline $100-125$ & 13.25 & 5.12 \\
\hline $125-150$ & 11.6 & 0.48 \\
\hline
\end{tabular}

The dynamic modelling for pitch control of the vehicle may be realized with the help of Fig 7. AUV150 performs heave operation with the help of a pair of vertical tunnel thrusters, which can pump out column of water from the water layer lying immediately underneath. This results in a reaction force $\mathrm{F}$ exerted by the water column instead as a result of which the vehicle displaces along the $\mathrm{Z}$ axis. However in the case of pitching, a couple $\tau$ acts on the vehicle about the $Y$ axis. Considering the location of $\mathrm{CG}$ at the geometric centre of the vehicle, the moment thus generated can be expressed as follows:

$$
\tau=F \cos (\theta t) \times L \cos (\theta t)
$$

Where,

$\theta t$ is the pitch angle at instant $t$
$\mathrm{L}$ is the midway axial distance between the two tunnel thrusters. This finally leads to the definition of the dynamic model for pitching as follows:

$$
\tau=I_{Y Y} \times\left(d^{2} \theta_{t} / d t^{2}\right)
$$

Where,

$\left(d^{2} \theta_{t} / d t^{2}\right)$ is the angular acceleration about $\mathrm{Y}$ axis.

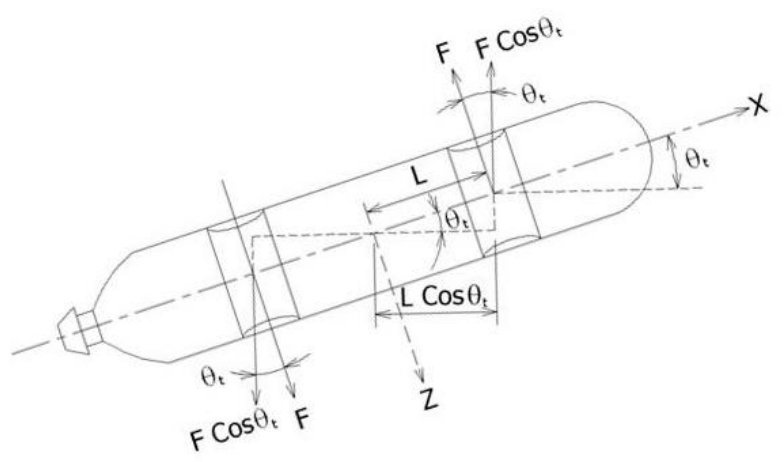

Fig. 7: Resolution of forces and development of couple for pitching of AUV

Figs. 8 and 9 respectively illustrate MatLab/ Simulink models for AUV-150 as regards pitch and linear velocity control.

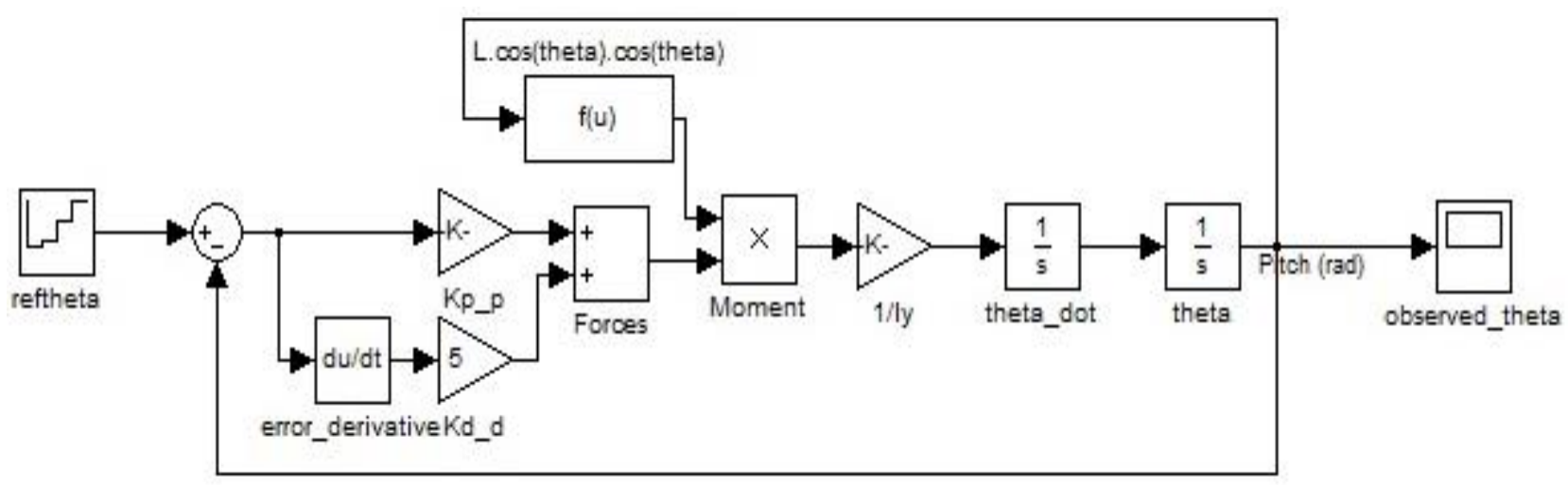

Fig. 8: Model towards simulation of pitch control for AUV-150

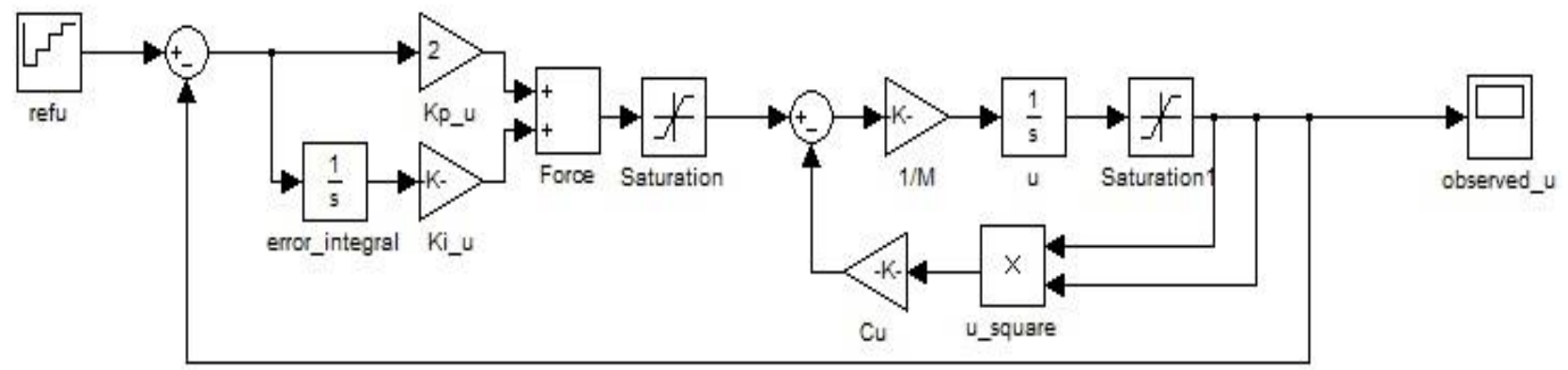

Fig. 9: Model towards simulation of velocity control for AUV-150 


\subsection{Modeling Environment Including Sea-Current and Resident Energy}

As for the data collected by AUV-150 during sea experimentations, it has been well observed that surface currents are rather insubstantial in strength. However, swells are more critical to the vehicle's stability during surface operations. On the contrary the entire body of observations are interesting enough with increase in depth. Table II summarizes the stochastic nature of the sea-currents as measured by the ADCP over a prolonged period of time on a daily basis. The values are represented in knots. Fig 10 (top) is illustrative of an approximated sea-current model plotted with values coming from a random process designed with parameters varying as a function of depth. Finally, the curve is smoothened out using a high-ordered polynomial fitting, which preserves the intrinsic variability of sea-current magnitude. It may well be seen that the magnitude is maximu $m$ for depths between 100 and 125 meters. The model is finally used for simulation of environment for validating effectiveness of the proposed planner, results of which shall be described in the subsequent section.
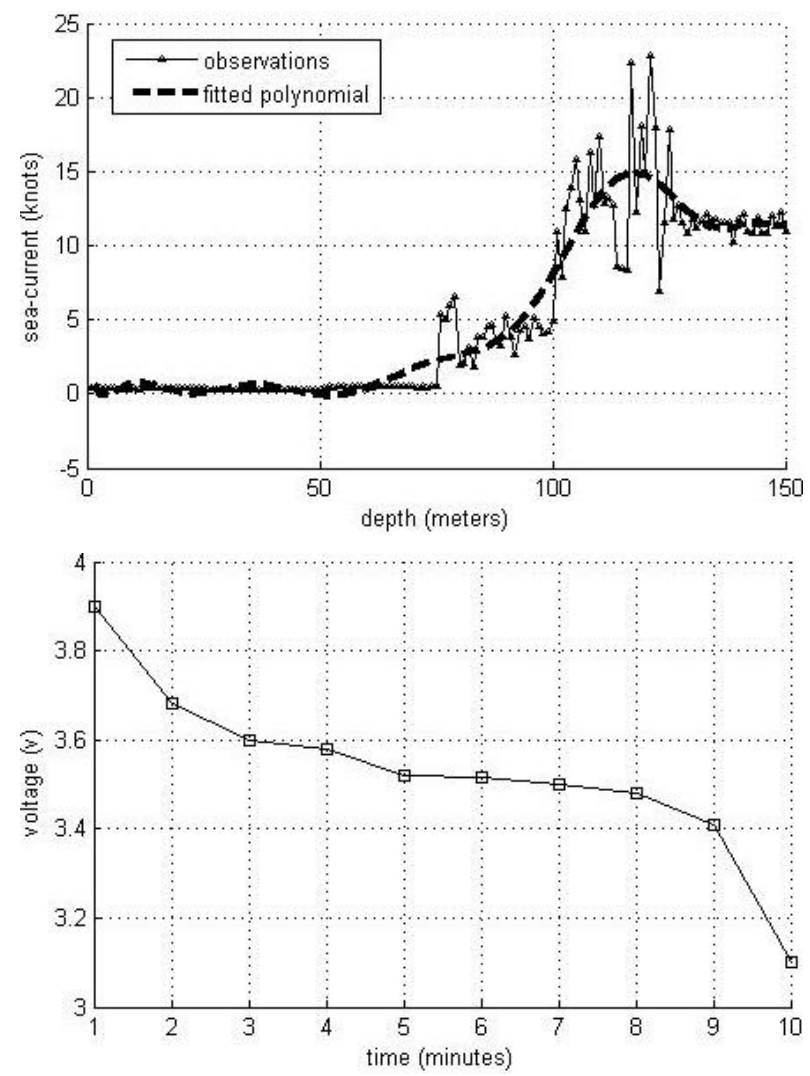

Fig. 10: Polynomial fitted curve characteristic of the observations of sea-current (top); typical discharge characteristic for Li-Po cells (bottom)

Fig. 10 (bottom) showcases the typical discharge characteristics of a Lithium Polymer (LiPo) cell. Discharge rate is quite nonlinear at the start as well as towards the end, with an approximate linear portion over a prolonged period. The characteristic acts as reference for simulating energy status and a model is developed for determining residual energy available with the systemat a given instant of time.

Let us assume $E$ to be the maximum energy available to the system, $e_{k}$ as the estimated energy at the time instant. Considering $V_{k}, i_{k}$ to be the instantaneous voltage and current at $t_{k}$, with $E_{\text {avg }}$ and $E_{\text {res }}$ as average energy and residual energy at $t_{k}$, following equations can be formulated:

$$
\begin{aligned}
& e_{k}=V_{k} \times i_{k} \\
& E_{\text {avg }}=(1 / k) \times \sum_{s=1}^{k} \\
& E_{\text {res }}=E-E_{\text {avg }}
\end{aligned}
$$

Finally for input at the fuzzy planner, the measure as computed using equations (6)-(8), is normalized as follows:

$$
E_{\text {norm }}=\left(E_{\text {res }} / E\right) \times 100
$$

The simulation environment includes FISPLAN integrated with pitch and velocity controllers as described earlier in the present section. Crisp observations are fed into the FISPLAN which infers the situation with the help of the fuzzy rule base and triggers the pitch and surge controllers accordingly with the corresponding reference values. Fig. 11 shows the Simulink block schematic for the overall control framework.

\section{Simulation Results \& Discussion}

The step-time for fuzzy planner is kept in consistence with the response-time of the controllers. Figs. 12 and 13 respectively are well illustrative of the fact that both the pitching and velocity controllers are rather sluggish in driving the system to the desired configuration within a time frame of 2 seconds. The controllers are quite responsive towards low values of reference whereas fail to reach the steady state for larger values of reference. By giving reference inputs to the controllers at an interval of 5 seconds steady state behaviour for the controllers is achieved. Hence, the fuzzy planner is invoked at an interval of 10 seconds which is represented in fig. 14. Fig. 15 illustrates as to how pitching and surge-velocity of the vehicle varies in response to the commands issued by the planner between successive planner intervals. Fig. 16 is representative of the situation when the AUV falls into different sea-current/depth zones. Fig. 16 (top) shows that the sea-currents are quite significant for the period $\mathrm{t}=1.5$ seconds, after which it drastically reduces. Fig. 16 (bottom) illustrates the fast change in depth of the vehicle from 150 to 100 meters of depth over the duration of first 1.5 seconds. Subsequently the vehicle soars with a lesser gradient up 90 meters of depth. This 
is evident of the fact as to how the planner responds to variations of sea-current on the basis of current depth zone, and how the system is restored to its zero-pitch configuration which is again showcased in fig. 17 wherein pitch and velocity sharply reduce to low values in response to reduced sea-currents.

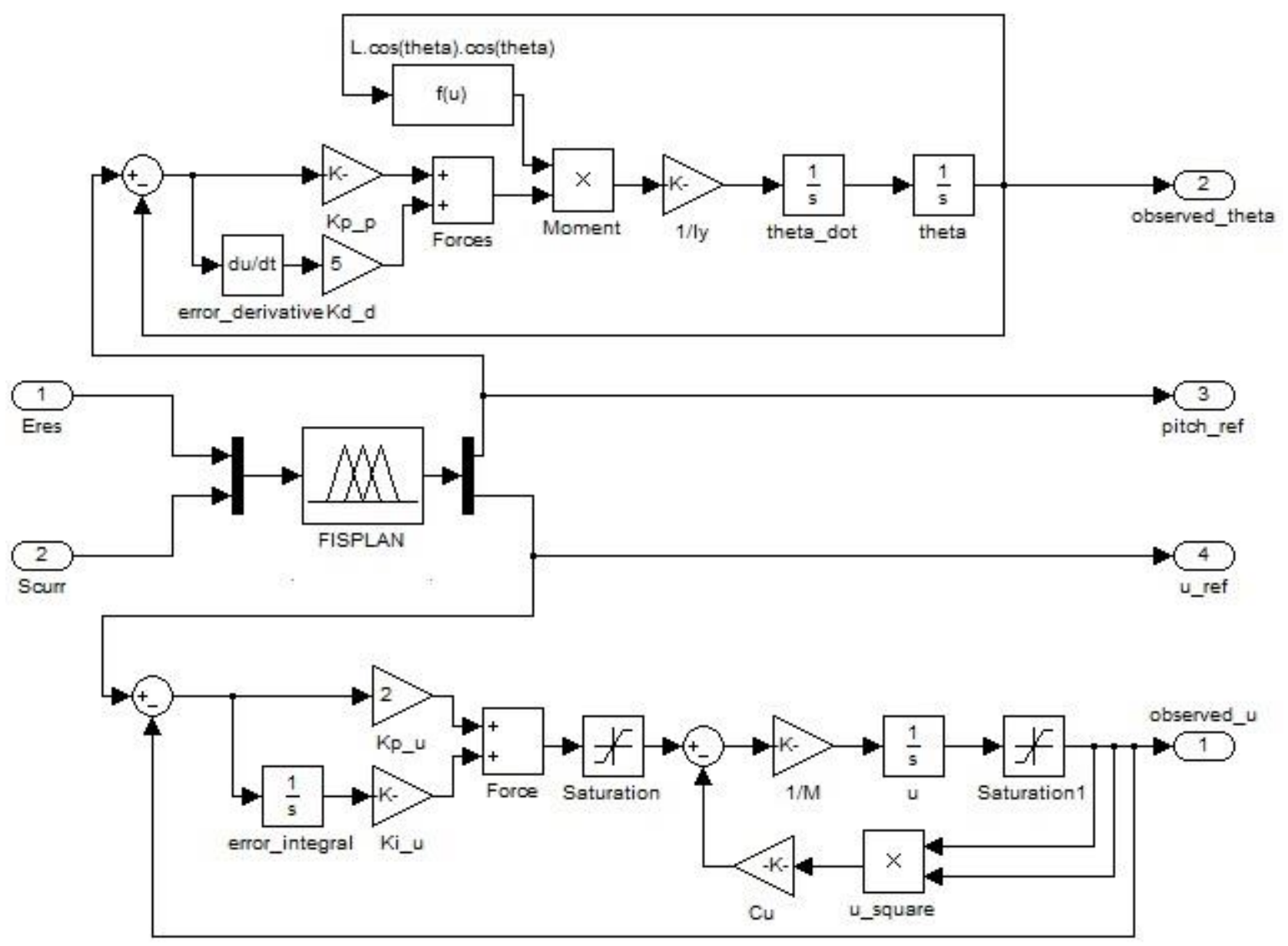

Fig. 11: Simulink model showing the FISPLAN working in coordination with the controllers
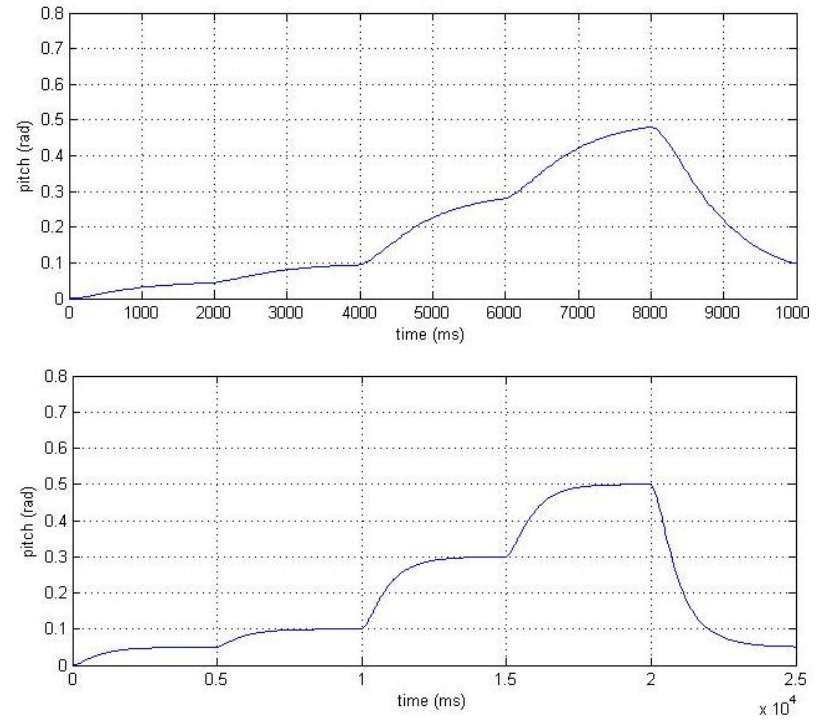

Fig. 12: Response time of pitch controller for staircase inputs having 2 seconds (top) and 5 seconds (bottom) sampling time
However, the planner reacts differently when both sea-currents as well as residual energy vary abruptly. Fig. 18 (top) plots the simulated residual energy given as a staircase input at interval of 50 seconds. Since the planner works at an interval of 10 seconds, it can be sufficiently reactive within the time frame of actual change in the observation of residual energy. Consequently, the vehicle pitches and soars up to a depth of 105 meters in response to the magnitude of sea-currents as has been previously illustrated in fig. 16 (top).

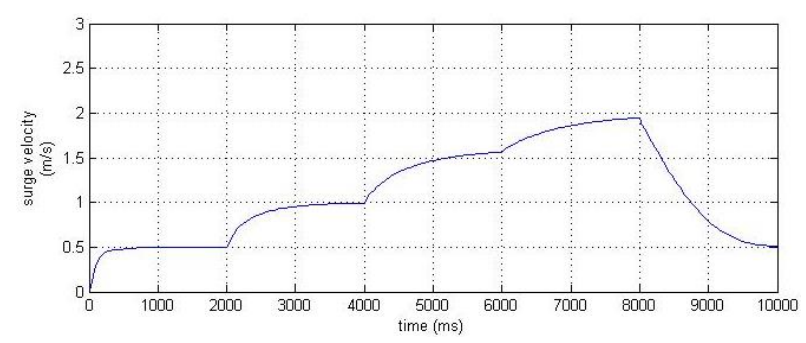




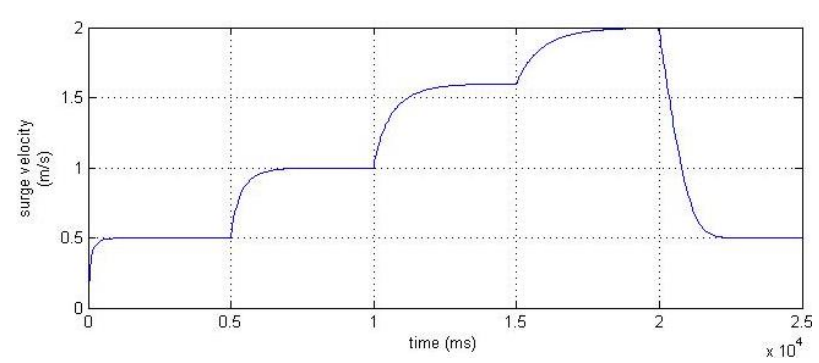

Fig. 13: Response time of velocity controller for staircase inputs having 2 seconds (top) and 5 seconds (bottom) sampling time

Subsequently the vehicle reaches a depth of close to 100 meters in comparison to the 90 meters of depth reached wherein residual energy was deliberately set at a constant high value. This evidently establishes the fact that the planner is effective in prioritising situations wherein low resident energy is considered to be more crucial than facing strong currents and the vehicle tries to push up with high pitching and low velocity. The latter is made clear in fig 19 , which shows a high pitching as done by the vehicle at $\mathrm{t}=0.5$ seconds whereas the surge velocity is low, which is representative of intelligence as imbibed by Rules 4-9 of FISPLAN.
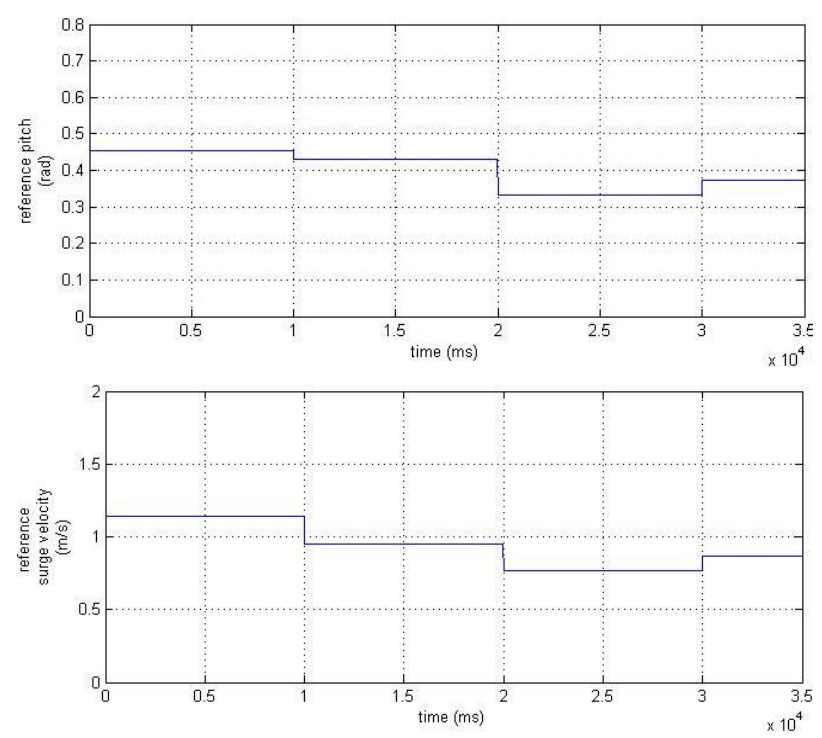

Fig. 14: Reference pitch (top) and velocity (bottom) as computed by fuzzy planner at a sampling rate of 10 seconds

\section{Conclusion}

The growing demand for autonomous underwater vehicles (AUVs) as effective technological means towards unmanned underwater explorations has given rise to an extensive research on effective control and planning software architectures. Starting with the originally classified reactive, deliberative and hybrid paradigms, control architectures for AUVs have evolved a lot to a very recent approach as to what may be defined as situation awareness, which makes the vehicle decisive about its present situations thereby dealing with circumstances instead of a more rigid scripted autonomy framework. In this context, the present paper proposes FISPLAN as a fuzzy logic reactive planning and control framework targeted towards escape operations in critical situations as regards facing strong sea-currents or running out of resident energy. The proposed architecture has been tested against a simulated situation for a real AUV prototype and actual environ mental parameters recorded during its field trials. Dynamics of the system are modelled and controller responses illustrated. Choice of planning interval is expressed as a function of the controllers' response. From an overall perspective, the work as presented in the paper bears significance in the context of decision against uncertainty for safe and energy optimal mission executions by AUVs. Perception of the current situation is of utmost importance wherein AUVs are deployed in completely unknown regions of the sea without any prior information about the characteristics of subsea currents. As a matter of future scope the planner may be augmented with a collision avoidance module and a global planner which can provide a further goaloriented escape decision in collaboration with the present reactive framework.
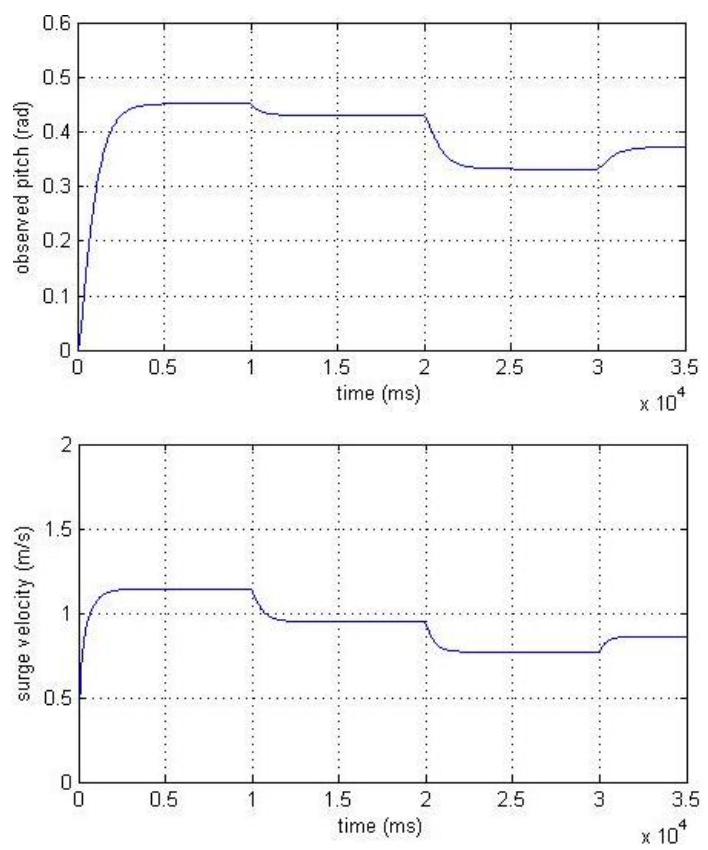

Fig. 15: Controller response for pitch (top) and surge velocity (bottom) to the planning time-varying commands issued by the planner

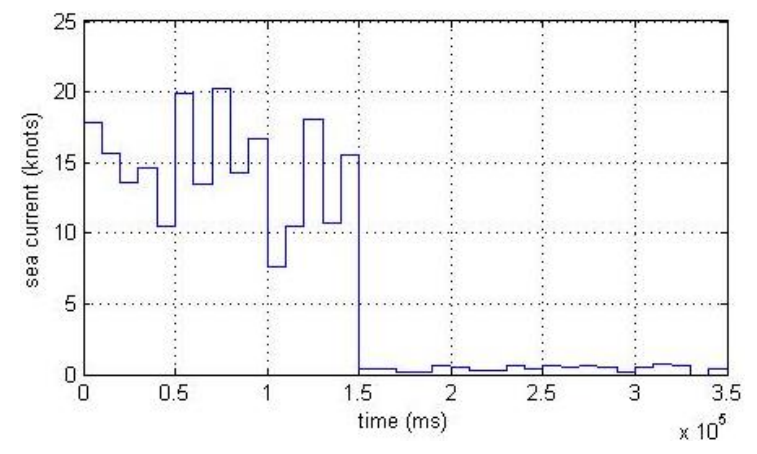

I.J. Intelligent Systems and Applications, 2013, 09, 47-57 


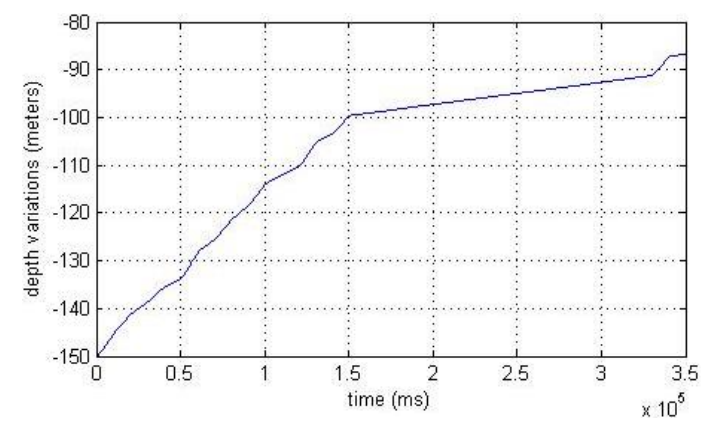

Fig. 16: Plot for simulated sea-current (top); variations in depth (bottom) as a consequence of reactive planning concerning seacurrent alone
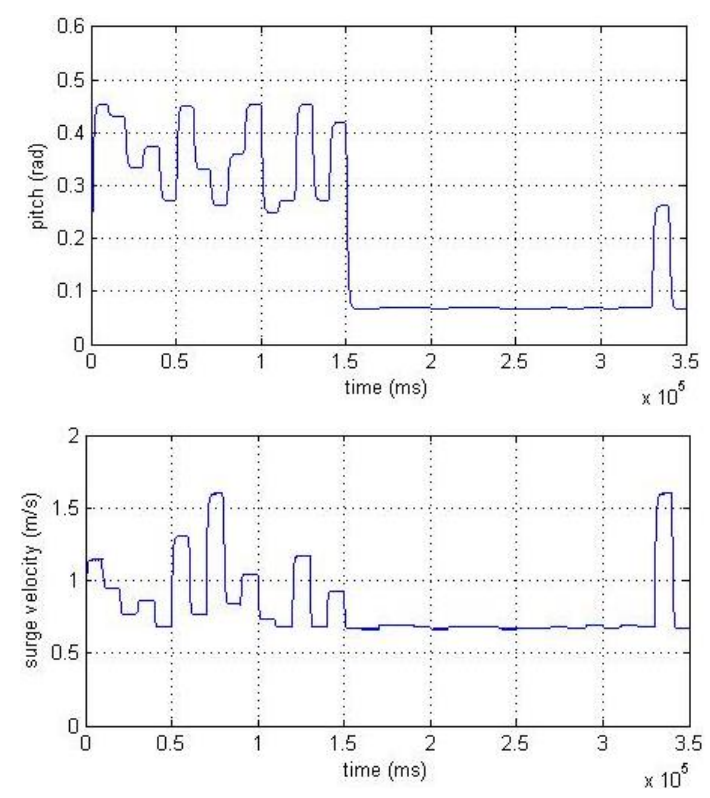

Fig. 17: Controller response for pitch (top) and velocity (bottom) as commanded by the planner concerning sea-current
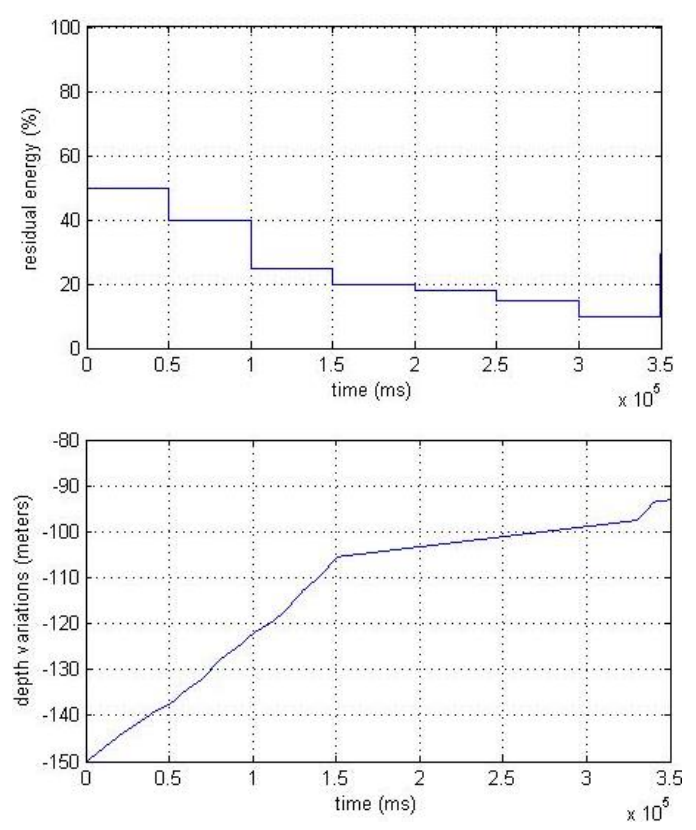

Fig. 18: Plot for simulated residual energy (top); variations in depth (bottom) as a consequence of reactive planning concerning both seacurrent as well as energy
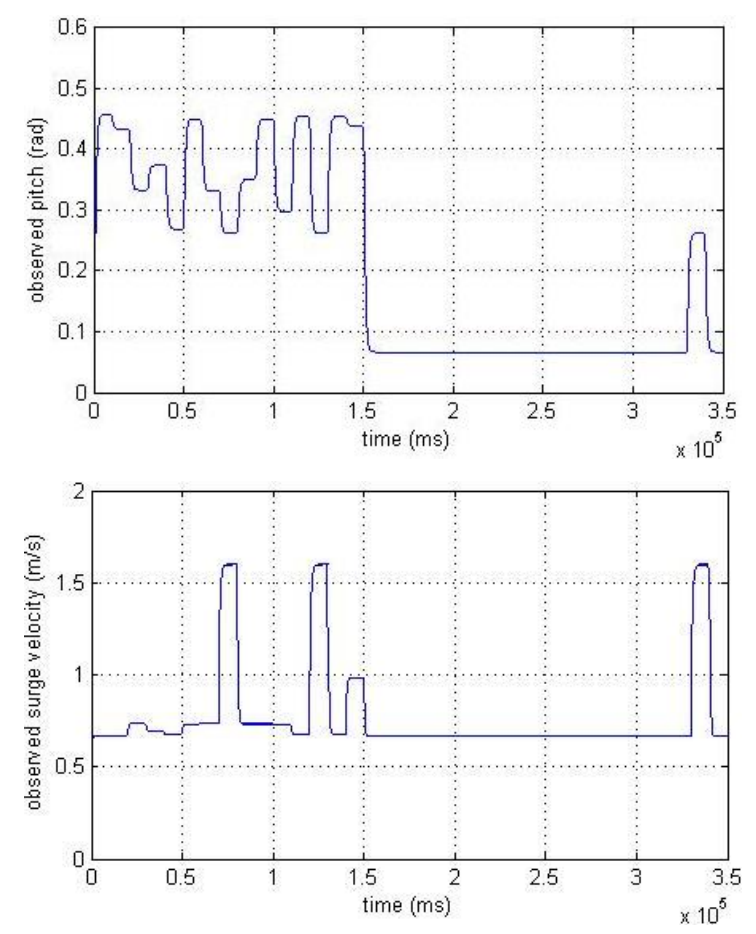

Fig. 19: Controller response for pitch (top) and velocity (bottom) as commanded by the planner concerning both sea-current as well as energy

\section{Acknowledgments}

The work as presented in the context of the present paper appertains to an extension of the activities of the Development of Autonomous Underwater Vehicle AUV-150, sponsored under a program by the Ministry of Earth Sciences (MoES), Govt. of India. The authors would like to thank the Project Development Group of CSIR-CMERI for approving and facilitating the extension activities in the form of an Institute Project. The authors would also acknowledge the efforts of the entire team for making this empirical work meaningful.

\section{References}

[1] J. YUH, Design and Control of Autonomous Underwater Robots: A Survey, Autonomous Robots 8, 7-24 (2000).

[2] Bellingham, J. G., Consi, T.R. and Beaton, R. M. Keeping layered control simple, Proceedings of the IEEE Symposium on Autonomous Underwater vehicle Technology, Washington D.C, USA, published as IEEE Catalog $\mathrm{N}^{\circ} 90 \mathrm{CH} 2856-3$, pp. 38, 1990.

[3] Zheng, X. Layered Control of a Practical AUV. Proceedings of the IEEE Symposium on Autonomous Underwater Vehicles Technology, Washington D.C., USA, pp. 142-147, 1992.

[4] Payton D.W., Keirsey D., Kimble D., Krozel J. and Rosenblatt K. Do Whatever Works: A Robust 
Approach to Fault-Tolerant Autonomous Control., Journal of Applied Intelligence, Vol. 2, pp.225250, 1992.

[5] Boswell A.J. and Leaney J.R., Using the subsumption architecture in a autonomous underwater robot: expostulations extensions and experiences, International Advanced Robotics program, Workshop on Mobile Robots for Subsea Environments, Monterey California, USA, 1994.

[6] Duane T. Davis, Thesis on Precision Control and Maneuvering of the Phoenix Autonomous Underwater Vehicle for entering a recovery tube, NA VAL POSTGRA DUATE SCHOOL Monterey, California, 1996.

[7] Fujii T., and Ura T, Development of an Autonomous Underwater Robot 'Twin-Burger' for Testing Intelligent Behaviors in Realistic Environments, Autonomous Robots, Vol. 3, No. 3, pp. 285-296, 1996.

[8] Rosenblatt J., Williams S., Durrant-Whyte H., Behavior-Based Control for Autonomous Underwater Exploration, IEEE International Conference on Robotics and Automation, San Francisco, USA.

[9] P. Ridao, J. Yuh', J. Batlle, K. Sugiharat, On AUV Control Architecture, Proceedings of the IEEE/RSJ International Conference on Intelligent Robots and Systems, 2000.

[10] Conor McGann, Frederic Py, Kanna Rajan, John Ryan, Richard Henthorn, Adaptive Control for Autonomous Underwater Vehicles, http://www.aaaipress.org/Papers/AAAI/2008/AAA I08-209.pdf

[11] C. Lin, S. Ren, X. Feng, Y. Li and J. Xu, Autonomic Element Based Architecture for Unmanned Underwater Vehicles, Proceedings of OCEANS 2010 IEEE - Sydney, Page(s): 1 - 5 .

[12] T. B. Koay, Y. T. Tan, Y. H. Eng., R. Gao, M. Chitre, J. L. Chew, N. Chandhavarkar, R. R. Khan, T. Taher, J. Koh, STARFISH-A s mall team of Autonomous Robotic Fish, Indian Jr. of GeoMarine Sciences, Vol. 40(2), April 2011, pp. 157167.

[13] Andres el-Fakdi, PhD Thesis on Gradient-Based Reinforcement Learning Techniques for Underwater Robotics Behavior Learning, University of Girona, 2010.

[14] ZEYN A SAIGOL, PhD Thesis on Automated Planning for Hydrothermal Vent Prospecting using AUVs, University of Birmingham, 2011.

[15] Emilio Miguela'n ez, Pedro Patro'n, Keith E. Brown, Yvan R. Petillot, and David M. Lane, Semantic Knowledge-Based Framework to Improve the Situation Awareness of Autonomous Underwater Vehicles, IEEE TRANSACTIONS
ON KNOWLEDGE AND DATA ENGINEERING, VOL. 23, NO. 5, MAY 2011.

[16] Subrata Das, High-Level Data Fusion, ARTECH HOUSE, INC. 2008, ISBN-13: 978-1-59693-281-4, pp: 6-7.

[17] L.A. Zadeh, Fuzzy sets, Information and Control 8 (1965) 338-353.

[18] E.H. Mamdani, S. Assilian, Application of fuzzy algorithms for control of simple dynamic plant, Proceedings of the Institute of Electrical Eng ineers 121 (1974) 1585-1588.

\section{Authors' Profiles}

Subhra Kanti Das (1982,-) male, West Bengal, India, Scientist, received the Bachelor of Engineering in Computer Science and Engineering from University of Kalyani, West Bengal, India in 2004. He is a PhD scholar at department of CSE in University of Jadavpur, West Bengal, India. His research interests include probabilistic measures towards navigation and localization of underwater mobile robotic systems, and application of AI techniques in development of control software architecture. He is the recipient of Young Engineer Award from Institution of Engineers India (IEI) in 2011.

Dibyendu Pal (1978,-) male, West Bengal, India, Scientist, received the Bachelor of Engineering in Electronics and Communications Engineering from University of Kalyani, West Bengal, India in 2000. He is a $\mathrm{PhD}$ scholar at department of ECE in University of Jadavpur, West Bengal, India. His research interests include adaptive robust control techniques and application of Fuzzy logic based model linearization to autonomous control of unmanned underwater vehicles. $\mathrm{He}$ is one of the authors for the copyright of control software of intelligent mobile robotic system IRS developed by CSIR-CMERI.

How to cite this paper: Subhra Kanti Das, Dibyendu Pal,"Formulation of FISPLAN: A Fuzzy Logic based Reactive Planner for AUVs towards Situation Aware Control", International Journal of Intelligent Systems and Applications(IJISA), vol.5, no.9, pp.47-57, 2013. DOI: 10.5815/ijisa.2013.09.06 